\title{
Von Forschungsdaten und wissenschaftlichen Sammlungen
}

\author{
Zur Arbeit des Stakeholdergremiums „Wissenschaftliche Sammlungen“ in DARIAH-DE
}

DOI 10.1515/bfp-2016-0036

Zusammenfassung: Die Digitalisierung der Gesellschaft führt zu methodischen Veränderungen in den Geistes- und Kulturwissenschaften, die u. a. in einer Reflexion und Diskussion über die Nutzungsmöglichkeiten von digitalen Forschungsdaten und Wissenschaftlichen Sammlungen führt. Im Rahmen von DARIAH-DE wurde aus diesem Grund das Stakeholdergremium „Wissenschaftliche Sammlungen“ gegründet, das sich mit methodischen und epistemologischen Praktiken in den Digital Humanities beschäftigt. Vor dem Hintergrund der zunehmenden Digitalität in der Forschung hat sich das Stakeholdergremium als Aufgabe gesetzt, einen Beitrag zur Schärfung des Selbstverständnisses der Digital Humanities und der Arbeiten von DARIAH-DE im Kontext von Forschungsdaten und Wissenschaftlichen Sammlungen zu leisten.

Schlüsselwörter: Forschungsdaten; epistemologische Praktiken; wissenschaftliche Sammlungen; DARIAH-DE; Stakeholdergremium

\section{Research Data and Scholarly Collections in the Arts and Humanities. Activities of the DARIAH-DE Stakeholder Committee „Wissenschaftliche Sammlungen“}

Abstract: The digitization of society results among other things in methodical changes in the Arts and Humanities, leading to reflection and discussion regarding new potential uses for digital research data and scientific collections. Within DARIAH-DE this subject - changing methodical and epistemological practices - has been discussed by the stakeholder committee for scientific collections. In the light of the spreading digitization of research the stakeholder committee commits itself to sharpen the self-conception of the Digital Humanities and the work of DARIAH-DE in the context of research data and scientific collections.

*Kontaktperson: Jenny Oltersdorf, oltersdorf@fh-potsdam.de Stefan Schmunk, schmunk@sub.uni-goettingen.de
Keywords: Research data; epistemological practices; scientific collections; DARIAH-DE; stakeholder committee

\section{Einleitung}

Die Digitalisierung der Gesellschaft ist ein Prozess, der vor über vierzig Jahren begonnen und in den letzten Jahren an großer Dynamik gewonnen hat. Digitale Technologien haben eine Vielzahl von Lebensbereichen nachhaltig verändert. $\mathrm{Zu}$ denken ist hier beispielsweise an die derzeit stattfindenden Transformationsprozesse in der Wirtschaft, die sich u.a. in zukunftsweisenden Technologiefeldern wie Produktengineering, Logistik, Service Robotik oder neuen Konzepten wie Industrie $4.0^{1}$ niederschlagen. $\mathrm{Zu}$ denken ist aber auch an Veränderungen im privaten Leben und vor allem den Wandel und die Neuorientierung in vielen Bereichen von Wissenschaft und Forschung.

Digitale Technologien verändern sozio-ökonomische Strukturen nachhaltig. Sie sind aus unserer Welt nicht mehr wegzudenken und führen zu grundsätzlichen Modifikationen der Lebens- und Arbeitswelt. Dieser Prozess ist in der Menschheitsgeschichte singulär und zugleich in seiner derzeitigen Breiten- und Tiefenwirkung sowie der Geschwindigkeit einzigartig. ${ }^{2}$ Vor allem in Bezug auf die Forschung hat die Digitalität ${ }^{3}$ dazu geführt, dass in den vergangenen fünf bis zehn Jahren (auch) in den Geistesund Kulturwissenschaften vermehrt methodologische Diskussionen begonnen und die eigenen epistemologischen Praktiken hinterfragt wurden. Die neuen technologischen Möglichkeiten führen zu veränderten Erkenntnisgegen-

1 Vgl. exemplarisch Sendler et al. (2013).

2 Vgl. Braunberger (2014).

3 Der Begriff Digitalität wird erst seit einigen wenigen Jahren verwendet, bezeichnet aber gerade die mannigfaltigen und zum Teil überlappenden und zeitgleich stattfindenden gesellschaftlichen Prozesse, die unmittelbaren Einfluss auch auf die Forschung haben. Im Rahmen der DFG wurde dieser Begriff aufgegriffen und ab 2016 soll eine Symposienreihe mit dem Titel „Digitalität in den Geisteswissenschaften“ die nur hier kurz skizzierten Themen diskutiert und analysiert werden. Siehe: http://digitalitaet-geisteswissenschaften.de. 
ständen und neuem Erkenntnisinteresse. ${ }^{4}$ Der Umgang mit digitalen Daten, Methoden ${ }^{5}$ und Forschungsinfrastrukturen und deren kritische Reflexion wird in den geistes- und kulturwissenschaftlichen Feldern unter dem Begriff „Digital Humanities“ zusammengefasst.

Die interdisziplinäre und interinstitutionelle Auseinandersetzung mit dem Thema wissenschaftliche Sammlung und Forschungsdaten vor dem Hintergrund zunehmender Digitalität ist bislang ein Desiderat, das durch das Stakeholdergremium „Wissenschaftliche Sammlungen“ aufgegriffen und diskutiert wird. Der bewusst weite Blickwinkel des Gremiums umfasst sowohl die Perspektive von Einzelforschern, Gedächtnisorganisationen und (Forschungs-)Infrastrukturanbietern.

Im Rahmen des vorliegenden Artikels wird das Spektrum der relevanten Themen aus Sicht des Stakeholdergremiums „Wissenschaftliche Sammlungen“ diskursartig vorgestellt. Ziel ist es, Fragestellungen und ausgewählte Ergebnisse des Gremiums zu präsentieren. Vor dem Hintergrund der zunehmenden Digitalität in der Forschung versteht sich die Auseinandersetzung mit wissenschaftlichen Sammlungen durch das Gremium vor allem als Beitrag zur Schärfung des Selbstverständnisses der Digital Humanities und als begleitende wissenschaftliche Arbeit im Projekt DARIAH-DE.

\section{Das Stakeholdergremium „Wissenschaftliche Sammlun- gen“}

In der 2. Phase des Projekts DARIAH-DE (2014-2016) wurde das Stakeholdergremium „Wissenschaftliche Sammlungen“ ins Leben gerufen. ${ }^{6}$ Im Gremium sind Wissenschaftler aus unterschiedlichen Geistes- und Kulturwissenschaften vertreten. Sie repräsentieren Universitäten, außeruniversitäre Forschungseinrichtungen, Akademien und Gedächtnisorganisationen. Die interdisziplinäre und institutionell breit gefächerte Zusammensetzung ermöglicht einen disziplinen- und institutionenübergreifenden Austausch, der neue methodische, interdisziplinäre und zugleich fachspezifische Blickwinkel eröffnet.

Ziel des Gremiums ist es, grundsätzliche Fragen der Generierung, Langzeitnutzung und Verfügbarkeit sowie der Analyse von geistes- und kulturwissenschaftlichen di-

4 Vgl. Gold (2012).

5 Reiche et al. (2014).

$6 \mathrm{https}$ //de.dariah.eu/stakeholdergremium-wissenschaftliche-sam mlungen. gitalen Forschungsdaten und digitalen wissenschaftlichen Sammlungen zu diskutieren. Die identifizierten Schwerpunktthemen reichen von einer benötigten (Arbeits-)Definition des Begriffs wissenschaftliche Sammlung über technische Anforderungen bis hin zu juristischen Fragen in Bezug auf wissenschaftliche Sammlungen und digitale Forschungsdaten. Als eine der größten Herausforderungen im Umgang mit Sammlungen hat das Gremium die mangelnde Sichtbarkeit und Auffindbarkeit benannt, die dazu führt, dass bereits digital vorliegende Sammlungen und ihre Metadaten nicht bzw. kaum für Forschungsvorhaben berücksichtigt werden können. Neben der Diskussion dieser fachwissenschaftlichen Fragen ist es eine weitere Aufgabe des Gremiums, sich kritisch mit den inhaltlichen und entwicklungsspezifischen Aufgaben von DARIAH-DE auseinanderzusetzen und die Ergebnisse dieser Diskussionen in das Projekt zurückzuspiegeln. Der unmittelbare Austausch zwischen Nutzern der DARIAH-DE Infrastruktur und dem Projekt selbst ist dadurch bestmöglich gewährleistet.

\section{Themencluster}

Durch das Stakeholdergremium „Wissenschaftliche Sammlungen“" wurden eine Reihe von Themen identifiziert und diskutiert. Eine komprimierte Auswahl soll im Folgenden skizzenhaft präsentiert werden.

Eine der ersten Aufgaben des Gremiums war die Erarbeitung einer (Arbeits-)Definition für die in den Geistesund Kulturwissenschaften neuen und zugleich zentralen Begriffe Forschungsdaten und wissenschaftliche Sammlungen. Die Schwierigkeit eines definitorischen Zugangs zu dem Begriff Forschungsdaten wird beispielhaft im DARIAH-DE Working Paper „Diskussion und Definition eines Research Data LifeCycle für die digitalen Geisteswissenschaften"7 erläutert. Die Frage nach der Definition des Begriffs Sammlung wird in der einschlägigen Forschungsliteratur ${ }^{8}$ vielfach aufgeworfen - zum Teil sogar bereits im Titel. So fragen sich beispielsweise James Curall, Micheal Moss und Susan Stuart im Jahr 2004 ,What is a collection?" 9

Sie umfassend zu beantworten ist jedoch nicht trivial. Aufgrund der Vielzahl verschiedener Einrichtungen und Interessengruppen sowie deren heterogenen Ansprüche an Sammlungen und den Umgang mit ihnen, ist es eine große Herausforderung, eine konsensfähige Definition

7 Puhl et al. (2015).

8 Vgl. dazu beispielsweise Palmer et al. (2006), Logoze und Fielding (1998) und Lee (2005).

9 Curall, Moss und Stuart (2004). 
herbeizuführen. „The three international organizations ICA, IFLA and ICOM have never engaged in any common definition of what a collection is." ${ }^{\text {"10 }}$

Auch im Gremium zeigte sich deutlich, dass die Definitionsmerkmale je nach Fachdisziplin und Hintergrund stark divergieren. Insbesondere die Perspektive von Gedächtnisorganisationen und die Forscherperspektive stehen sich nicht nur in der Literatur sondern auch im Gremium häufig diametral gegenüber. Die Bedürfnisse dieser beiden Gruppen im Hinblick auf wissenschaftliche Sammlungen und Forschungsdaten sind heterogen, vor allem bezüglich rechtlicher, kuratorischer und nutzungsspezifischer Fragen. Besonders deutlich wird die Heterogenität des Blickwinkels bei der Frage nach den Motiven für das Erstellen einer Sammlung. Digitale, wissenschaftliche Sammlungen können, je nach Perspektive, allein aufgrund einer wissenschaftlichen Fragestellung erstellt und entsprechend aufbereitet worden sein oder eher generischen Charakter haben, mithin an keinen wissenschaftlichen Entstehungskontext gebunden sein und allein durch die weitere Aufbereitung bzw. Annotations- und Anreicherungsmöglichkeiten wissenschaftlichen Charakter erhalten. Diese Aspekte fanden Eingang in die vom Stakeholdergremium „Wissenschaftliche Sammlungen“ erstellte Definition für Forschungsdaten:

„Daten, unabhängig ihrer Provenienz, werden im Kontext einer
geistes- und kulturwissenschaftlichen Forschungsfrage zu digi-
talen Forschungsdaten, sobald sie gesammelt, beschrieben, aus-
gewertet und/oder erzeugt und in maschinenlesbarer Form zum
Zwecke der Nachvollziehbarkeit von Forschungsergebnissen so-
wie zur Archivierung, Zitierbarkeit und Weiterverarbeitung auf-
bewahrt werden. Eine Aggregation von Forschungsdaten (n>1)
ist in diesem Sinne eine Wissenschaftliche Sammlung.“

Gerade für die Geistes- und Kulturwissenschaften ist solch eine Definition von grundlegender Bedeutung, wenngleich bislang in den einzelnen Fächern kein Konsens über eine Definition zu Forschungsdaten erzielt wurde..$^{12}$ Gedächtnisorganisationen sprechen bislang von Archiv- und Sammlungsgut, von Beständen, Nachlässen oder auch von Sammlungen, sofern es sich um eine Gruppe von physischen Objekten - beispielsweise Kunstwerken, archäologischen Artefakten etc. - handelt. Von den Forschenden selbst werden diese definitorischen Konzepte ebenfalls übernommen und zugleich um disziplinäre Begriffe er-

10 Wickett et al. (2013, S. 7).

11 Eine wissenschaftliche Sammlung muss aus mindestens zwei Forschungsdaten bestehen.

12 Vgl. exemplarisch für die Geschichtswissenschaft die Umfrage und Analyse von Andorfer (2015). gänzt. In der Geschichtswissenschaft wurde beispielsweise in den letzten 200 Jahren ein Methoden- und Theoriekanon etabliert, der zu den Begriffen „Überreste“, „Publikationen“ und „Quellen“ führte, um definieren zu können, ob es sich um physische Objekte, gedruckte (wissenschaftliche) Werke oder um zeitgenössisches originäres Quellenmaterial handelt. ${ }^{13}$ Gerade hier gilt es zu überprüfen, ob diese methodischen Konzepte durch die Veränderungen der Digitalität noch anwendbar sind. Zugleich muss sichergestellt werden, dass methodische disziplinäre Grundannahmen und Konzepte, wie beispielsweise das von Reinhard Koselleck 1977 formulierte „Vetorecht der Quellen" ${ }^{14}$ auf digitale Forschungsdaten und dementsprechende auch auf wissenschaftliche Sammlungen im Zeitalter der Digitalität angewandt werden können. Laut Koselleck „verbieten uns [Quellen], Deutungen zu wagen oder zuzulassen, die aufgrund eines Quellenbefundes schlichtweg als falsch oder als nicht zulässig durchschaut werden können. Falsche Daten, falsche Zahlenreihen, falsche Motiverklärungen, falsche Bewusstseinsanalysen: all das und vieles mehr lässt sich durch Quellenkritik aufdecken." ${ }^{15}$ Berücksichtigt man diese Grundvoraussetzung in der Geschichtswissenschaft, resultiert dies in der Erkenntnis, dass Forschungsdaten auch ihre Provenienz und die entsprechenden kontextualisierenden Informationen ihrer Entstehung mit abbilden müssen. Nur so ist letztlich die von Koselleck postulierte methodische Grundannahme nicht weniger als die Frage der Validität und Reliabilität von Forschungsergebnissen - abzubilden.

Das Stakeholdergremium hat aus diesem Grund in einem weiteren Arbeitsschritt folgende Definition für den Begriff der wissenschaftlichen Sammlung auf Basis der Definition zu Forschungsdaten erstellt, die wie folgt lautet:

Eine wissenschaftliche Sammlung relevanter digitaler Forschungsdaten

- besteht aus diskreten, voneinander verschiedenen, logisch unabhängigen Einheiten (Bilderregel),

- muss nicht aus einem Forschungsanliegen heraus entstanden sein, ist aber Gegenstand wissenschaftlicher Fragestellungen bzw. hat das Potential zu einer wissenschaftlichen Analyse und dient der Validierung von Aussagen, Methoden, Thesen, Hypothesen oder

13 Eine Differenzierung, die in der Geschichtswissenschaft als Quellenlage und Quellenkritik bezeichnet wird und die trotz unzähliger methodischer Reflexionen und Veränderungen in ihrer Grundkonstruktion auf Johann Gustav Droysen zurückgeht und damit ein Konzept des 19. Jahrhunderts ist. Vgl. hierzu exemplarisch die Dissertation von Buller (2002).

14 Koselleck (1977).

15 Ebd. S. 47. Vgl. hierzu auch Jordan (2010). 
Theorien in Forschung und Lehre (Reproduzierbarkeit),

- kann sowohl Ursprung als auch Ergebnis wissenschaftlicher Arbeit sein (Research Data Life Cycle),

- ist in einer regelhaften Form maschinenlesbar, dokumentiert, idealerweise nach internationalen Standards erfasst und mit Normdaten ausgezeichnet (Prozessier- und Interpretierbarkeit),

- gibt Auskunft über ihren Rechtsstatus (z. B. Nutzungsbedingungen und Lizenzen),

- dient der Ordnung der Sammlungsgegenstände und der archivischen Sicherung (Archivierung).

In Abgrenzung zu Repositorien können digitale wissenschaftliche Sammlungen im Zusammenhang mit der Bearbeitung einer Forschungsfrage ad hoc gebildet werden. ${ }^{16}$ Bei einer traditionellen nicht-digitalen Sammlung besteht ein Unterschied zwischen physischen Sammlungsobjekten und dem Ort der Sammlung. Eine unter einer Bilderregel ad hoc generierte, aggregierte Sammlung aus unterschiedlichen digitalen Quellen in einem eigenen Repositorium kann andere Sammlungen enthalten und es ist offen, ob sie nach Nutzung bestehen bleibt oder wieder zerfällt. Nicht zuletzt ist nicht absehbar - ein Grundproblem, das $\mathrm{zu}$ allen Zeiten und auch in allen Entstehungskontexten schon immer bestand -, ob und welche Daten in Zukunft Forschungsrelevanz erlangen könnten. Aus diesem Grund kann auch keine Selektion oder Auswahl nach Relevanzkriterien vorgenommen werden.

Der Wert von digitalen wissenschaftlichen Sammlungen, so die These des Gremiums, wird aus diesen Gründen für die Forschung bislang unterschätzt. Es müssen technologische und informationswissenschaftliche Lösungen gefunden werden, um wissenschaftliche Sammlungen und Forschungsdaten leichter auffinden zu können und sie somit ins Blickfeld interessierter Wissenschaftler zu führen. Nur wenn dies gelingt, wird es letztlich möglich sein, dass die Daten- bzw. Quellenlage bei Forschungsvorhaben umfassend (und quellenkritisch) berücksichtigt werden kann.

Diese vom DARIAH-DE Stakeholdergremium „Wissenschaftliche Sammlungen“ vorgenommenen Definitionsansätze grenzen sich von einer Reihe bisheriger Definitionskonzepte ab. Der Wissenschaftsrat bezieht sich beispielsweise in den „Empfehlungen zu wissenschaftlichen Sammlungen als Forschungsinfrastrukturen"17 aus dem Jahr 2011 in seinen Ausführungen auf objektbasierte

16 Vgl. Begriffsdefinition zu Repositorien u.a. Pampel, Goebelbecker und Vierkant (2012).

17 http://www.wissenschaftsrat.de/download/archiv/10464-11.pdf.
Sammlungen, denen „durch ihre spezifische Materialität eine besondere Wirkung zu[kommt], die in Forschung und Lehre nutzbar gemacht wird." ${ }^{18}$ Der Terminus objektbasiert bezieht sich in diesem Kontext unmittelbar auf Sammlungen haptischer Gegenstände, die - so die Ausführungen in den Empfehlungen - noch nicht das volle Nutzungspotential erreicht haben. Begründet wird dies u. a. mit einer unzureichenden Erschließung, mangelnder Sichtbarkeit, Betreuung, Pflege oder Unterbringung der Sammlungen. ${ }^{19}$ Bezieht man die Veränderungen für die Geistes- und Kulturwissenschaften ein, die durch die zunehmende Digitalität entstanden sind bzw. entstehen, so muss eine Erweiterung des bisherigen Verständnisses von wissenschaftlichen Sammlungen erfolgen und diese nicht nur auf objektbasierte, physische Sammlungen bezogen werden, sondern auch als Aggregation von digitalen wissenschaftlichen Forschungsdaten und Sammlungen verstanden werden. Hierdurch wird nicht nur eine Öffnung des Begriffs erzielt, sondern zugleich der derzeitigen Forschungspraxis Rechnung getragen und die Möglichkeit geboten, neue methodische Konzepte im Zeitalter der Digitalität zu eröffnen.

Ein weiteres deutliches Unterscheidungsmerkmal in der Betrachtung wissenschaftlicher Sammlungen zwischen dem Stakeholdergremium und dem Wissenschaftsrat betrifft die externe Statusbestimmung einer Sammlung im Hinblick auf ihren wissenschaftlichen Nutzen. Der Wissenschaftsrat rät zu folgendem Vorgehen:

\footnotetext{
„Von grundlegender Bedeutung ist eine Statusbestimmung der wissenschaftlichen Sammlungen. Die vorhandenen Bestände sollten durch die unmittelbar für die Sammlungen verantwortlichen Personen bzw. Institutionen anhand sammlungsspezifischer, primär auf wissenschaftliche Nutzung und Nutzbarkeit gerichteter Bewertungskriterien erfasst und qualitativ eingeordnet werden. Dies erhöht die Transparenz und Sichtbarkeit der Sammlungen und ermöglicht eine realistische Wertschätzung.“
}

Hier vertritt das Stakeholdergremium „Wissenschaftliche Sammlungen" einen deutlich anderen Zugang. Eine externe Statusbestimmung wird in diesem Kontext eher als hinderlich verstanden, denn dadurch würden faktisch Sammlungen ausgeschlossen werden, die zu einem späteren Zeitpunkt oder auf der Basis vielleicht noch unbekannter technologischer Möglichkeiten oder veränderter Forschungsfragen relevant für die Forschung werden könnten. Das Kriterium der Wissenschaftlichkeit sollte aus Sicht des Gremiums nicht ausschließlich a priori vergeben

18 Ebd.

19 Ebd. 
werden. Eine Sammlung verdient nicht nur dann das Prädikat „wissenschaftlich“, wenn sie im Rahmen eines wissenschaftlichen Forschungsvorhabens entstanden ist und entsprechend bearbeitet wurde. Auch a posteriori kann eine Sammlung Wissenschaftlichkeit vorweisen, wenn sie zur Beantwortung einer wissenschaftlichen Fragestellung erschlossen und so in den Forschungsprozess eingebracht wird - eine wesentliche und wichtige Rolle, die die Gedächtnisorganisationen zukünftig vermehrt übernehmen könnten. Daraus folgt, dass ein sog. inklusionistischer Ansatz bei der Speicherung von Forschungsdaten und wissenschaftlichen Sammlungen angestrebt werden sollte. Inklusion, also das Speichern aller digitalen Forschungsdaten, ist hierbei ein pragmatischer und sinnvoller Ansatz, da sich die wissenschaftliche Community häufig selbst uneins darüber ist, welche Sammlungen erhalten werden sollen und welche nicht. Gerade im digitalen Bereich ist Inklusion heute fast ohne finanzielle Mehraufwendungen möglich. In einem ersten Schritt könnte dies durch eine reduzierte Sammlungsbeschreibung und eine entsprechend niedrigschwellige Infrastruktur geleistet werden. $\mathrm{Zu}$ denken wäre hier beispielsweise an ein zentrales Register für digitale wissenschaftliche Sammlungen in Deutschland. Sammlungsbeschreibungen könnten mit einem Kernset an Metadaten gespeichert und sichtbar gemacht werden. Die Nachnutzbarkeit von digitalen wissenschaftlichen Sammlungen, vor allem auch solchen, die nicht in einem unmittelbar institutionellen Forschungskontext entstanden sind, würde wesentlich erhöht werden.

Mit der Deutsche Digitale Bibliothe $\mathrm{k}^{20}$ und Europeana $^{21}$ existieren bereits Plattformen, auf denen zukünftig Sammlungen unter einer einheitlichen Suchoberfläche gebündelt werden können. Aufgrund der derzeitigen inhaltlichen Ausrichtung ist jedoch abzuwarten, inwiefern sie geeignete Lösungen für heterogene, digitale wissenschaftliche Sammlungen bieten, die bisher nicht in Gedächtnisorganisationen verzeichnet worden sind und bei denen es sich gerade nicht um originäres Sammlungsgut, Achivalien oder Publikationen handelt.

Ausgehend von dem Bemühen die Begriffe Forschungsdaten und wissenschaftliche Sammlungen zu schärfen und gegen andere Definitionsansätze abzugrenzen, identifizierte das Gremium die Rollenverteilung zwischen Forschenden, Gedächtniseinrichtungen und Universitäten bzw. Forschungseinrichtungen vor dem Paradigma der Digitalität als weiteren relevanten Diskussionspunkt.

20 https://www.deutsche-digitale-bibliothek.de/.

21 http://www.europeana.eu/.
Auch das Thema Sammlungskuration wurde als virulent eingestuft. ${ }^{22}$ In diesem Punkt sind Unterschiede im Hinblick auf digitale und physische Sammlungen besonders deutlich. In Gedächtnisorganisationen wie Bibliotheken und Archiven ist das Makulieren von physischen Objekten, je nach Sammelauftrag und strukturellen Rahmenbedingen, gängige Praxis. Es erfolgt eine permanente Bestandskontrolle entsprechend des Sammlungsauftrages bzw. den Nutzeranforderungen. Hat die Sammlungskontrolle bzw. Kassation von physischen Objekten in Gedächtnisorganisationen pragmatisch nachvollziehbare Gründe (Sammlungsauftrag, Platzmangel), erscheint das Löschen von bereits vorhandenen digitalen Daten im 21. Jahrhundert nicht mehr sinnvoll begründbar. Speicherkapazität ist heute weder aus technischer noch ökonomischer Sicht eine nennenswerte Herausforderung. Die Aufbewahrung von wissenschaftlichen Sammlungen, inklusive grundlegender Metadaten, stellt daher keine technologischen Schwierigkeiten (Speicherplatz, Bit Preservation etc.) mehr dar. Auch wenn digitale Sammlungen zu einem späteren Zeitpunkt technisch und/oder intellektuell nur schwer nutzbar sein sollten (veraltete Datei- und Datenformate etc.), so könnte argumentiert werden, dass ihre Darstellbarkeit bzw. Interpretierbarkeit im Rahmen von Forschungsprojekten erneut hergestellt, und die Einbindung in einen aktiven Forschungsprozess gewährleistet werden.

Das Thema Wahrnehmbarkeit bzw. Sichtbarkeit von Sammlungen berührt ebenfalls sehr eng rechtliche und lizenzspezifische Aspekte der Nutzung von wissenschaftlichen Sammlungen und Forschungsdaten. ${ }^{23}$ Das Spektrum rechtsrelevanter Themen umfasst neben klassischen, bibliotheksrechtliche Fragen, auch solche, die erst durch digitale Forschungsdaten und Methoden entstehen. Hier ist beispielsweise an den Bereich Text/Data Mining zu denken, der zukünftig neue juristische Fragestellungen für die Digital Humanities aufwerfen wird. Wenn alle Aggregations- bzw. Bearbeitungsstufen von Objekten einen schöpfe-

22 Einen ersten Anlaufpunkt bietet hier die Koordinierungsstelle für wissenschaftliche Universitätssammlungen in Deutschland. Siehe http://wissenschaftliche-sammlungen.de/de/.

23 Im Rahmen von DARIAH-DE wurde diese Thematik von Fachwissenschaftlern unterschiedlichster Fachdisziplinen sehr häufig angesprochen und eine rechtliche Unsicherheit bei der Nutzung und vor allem Publikation von Forschungsdaten artikuliert. Diese vermehrten Anfragen und das Bedürfnis nach weitergehenden Informationen zu dieser Thematik wurde von DARIAH-DE aufgegriffen und in Zusammenarbeit mit Dr. Paul Klimpel und John W. Weitzman (iRights.law) im Rahmen der DARIAH-DE Working Papers Juristische Handreichungen für die Nutzung von Forschungsdaten publiziert. Siehe Klimpel und Weitzmann (2015). 
rischen Wert darstellen, könnte dies u.U. dazu führen, dass sie zukünftig rechtlich abgesichert werden müssten.

\section{Perspektiven, Zukunft des Gremiums}

In der Folge dieses Beitrages sollen perspektivisch weitere Ergebnisse und Empfehlungen des Stakeholdergremiums veröffentlicht werden, um so die disziplinenübergreifende Diskussion zu den Themen Forschungsdaten, Kuration von Daten und wissenschaftliche Sammlungen $\mathrm{zu}$ stimulieren. Gerade vor dem Hintergrund digitaler Transformationsprozesse, denen sich insbesondere die Geistes- und Kulturwissenschaften nicht verschließen dürfen, ist es dringend notwendig, dass im Rahmen von Forschungsinfrastrukturvorhaben interdisziplinäre Diskussion- und Austauschplattformen geschaffen werden. Es ist daher eine zentrale Aufgabe, bei der Entwicklung digitaler Forschungsinfrastrukturen für die Geistes- und Kulturwissenschaften (beispielsweise durch DARIAH-DE und CLARIN-D ${ }^{24}$ ) Kompetenznetzwerke und soziale Infrastrukturen mit $\mathrm{zu}$ planen, sie aufzubauen und auf Dauer zu betreiben. Der Wissenschaftsrat hat dies bereits 2011 in seinen veröffentlichten „Empfehlungen zu Forschungsinfrastrukturen in den Geistes- und Sozialwissenschaften“ explizit gefordert. $^{25}$

\footnotetext{
„Als Orte der Begegnung regen solche sozialen Infrastrukturen in den Geisteswissenschaften durch ihre Programme Forschungsideen und -lösungen an, die üblicherweise erst zeitversetzt an den Heimatinstitutionen der eingeladenen Forscherinnen und Forscher in Forschungsergebnisse bzw. wegweisende Publikationen umgesetzt werden." 26
}

In diesem Sinne ist das Stakeholdergremium „Wissenschaftliche Sammlungen“ eine „soziale Infrastruktur“ und „Ort der Forschungskommunikation“, ${ }^{27}$ in dem disziplinund institutionsübergreifende Diskussionen ermöglicht werden, um aktuelle Fragestellungen zum Umgang, zu Nutzung und Speicherung von Forschungsdaten und wissenschaftlichen Sammlungen zu thematisieren. Dies soll in der dritten Phase von DARIAH-DE fortgeführt und inhaltlich ausgebaut werden. Für das Projekt DARIAH-DE ist dies von besonderer Bedeutung, da explizit eine interdisziplinäre Perspektive vertreten wird. Zugleich bietet das europäische Netzwerk von DARIAH-EU ${ }^{28}$ die Möglichkeit, diese Diskussionen in den europäischen Forschungsraum zu tragen und Diskussionen von Fachwissenschaftlern aus anderen europäischen Ländern einzubeziehen.

Die Diskussionen im Gremium haben gezeigt, dass in den Geistes- und Kulturwissenschaften ein großer Diskussionsbedarf im Hinblick auf methodische Reflexionen besteht, wenn es um digitale Forschungsdaten und wissenschaftliche Sammlungen geht. Die systematische, wissenschaftstheoretische Auseinandersetzung mit der eigenen Forschungspraxis erscheint besonders dann zielführend, wenn sie in enger Diskussion mit Mitarbeitern in den Gedächtnisorganisationen und (Forschungs-)Infrastrukturanbietern realisiert wird. Perspektivisch können so die fachspezifischen und oftmals hermetisch geschlossenen Diskussionen aufgegriffen und vielfältige Aspekte der Digitalität vergleichend, disziplinenübergreifend und in Kooperation mit Gedächtnisinstitutionen und Anbietern digitaler Infrastrukturen analysiert werden.

\section{Literaturverzeichnis}

Andorfer, Peter (2015): Forschungsdaten in den (digitalen) Geisteswissenschaften. Versuch einer Konkretisierung. (DARIAH-DE Working Papers 14). Göttingen: DARIAH-DE. Verfügbar unter urn: nbn:de:gbv:7-dariah-2015-7-2.

Braunberger, Gerald: Digitale Revolution. Macht der Maschinen. In: FAZ vom 27.04.2014. http://www.faz.net/aktuell/wirtschaft/me nschen-wirtschaft/digitale-revolution-macht-der-maschinen-12 910372.html.

Buller, Andreas (2002): Die Geschichtstheorien des 19. Jahrhunderts: Das Verhältnis zwischen historischer Wirklichkeit und historischer Erkenntnis bei Karl Marx und Johann Gustav Droysen. Berlin: Logos-Verlag.

Curall, J.; Moss, M.; Stuart, S. (2004): What is a collection? In: Archivaria, 58, 131-46.

Gold, Matthew K. (2012): Debates in the digital humanities. Minneapolis, Minn.: Univ. of Minnesota Press.

Jordan, Stefan (2010): Vetorecht der Quellen, Version: 1.0. In: Docupedia-Zeitgeschichte, 11.2.2010. Verfügbar unter https://docupe dia.de/zg/Vetorecht_der_Quellen.

Klimpel, Paul; Weitzmann, John H. (2015): „Forschen in der digitalen Welt. Juristische Handreichungen für die Geisteswissenschaften“. (DARIAH-DE Working Papers 12). Göttingen: DARIAH-DE. Verfügbar unter urn:nbn:de:gbv:7-dariah-2015-5-0.

Koselleck, Reinhart (1977): Standortbindung und Zeitlichkeit. Ein Beitrag zur historiographischen Erschließung der geschichtlichen Welt. In: Objektivität und Parteilichkeit in der Geschichtswissenschaft, hg.v. Reinhart Koselleck, Wolfgang J. Mommsen und Jörn Rüsen. München: Deutscher Taschenbuch-Verlag.

$24 \mathrm{http}: / /$ www.clarin-d.de/de/.

25 Wissenschaftsrat (2011).

26 Ebd. S. 68.

27 Ebd.

28 http://dariah.eu/. 
Lee, Hur-Li (2005): The Concept of Collection from the User's Perspective. In: The Library Quarterly, 75 (1), 67-85. DOI: 10.1086/ 428693.

Logoze, Carl; Fielding, David (1998): Defining collections in distributed digital libraries. In: D-Lib Magazine, 4 (11). Verfügbar unter h ttp://dlib.org/dlib/november98/lagoze/11lagoze.html.

Palmer, Carol L. et al. (2006): Collection Definition in Federated Digital Resource Development. In: $69^{\text {th }}$ Annual Meeting of the American Society for Information Science and Technology (ASIST). Austin: Richard B. Hill. Verfügbar unter http://eprints.rc lis.org/8638/.

Pampel, Heinz; Goebelbecker, Hans-Jürgen; Vierkant, Paul (2012): re3data.org: Aufbau eines Verzeichnisses von ForschungsdatenRepositorien. Ein Werkstattbericht. In: Mittermaier, Bernhard (Hg.): Vernetztes Wissen - Daten, Menschen, Systeme. Jülich: Zentralbibliothek, S. 61-74.

Puhl, Johanna; Andorfer, Peter; Höckendorff, Mareike; Schmunk, Stefan; Stiller, Juliane; Thoden, Klaus (2015): Diskussion und Definition eines Research Data LifeCycle für die digitalen Geisteswissenschaften. (DARIAH-DE Working Papers 11). Göttingen: DARIAH-DE. Verfügbar unter http://webdoc.sub.gwdg.de/pub/ mon/dariah-de/dwp-2015-11.pdf.

Reiche, Ruth; Becker, Rainer; Bender, Michael; Munson, Mathew; Schmunk, Stefan; Schöch, Christof (2014): Verfahren der Digital Humanities in den Geistes- und Kulturwissenschaften. (DARIAHDE Working Papers 4). Göttingen: DARIAH-DE. Verfügbar unter urn:nbn:de:gbv:7-dariah-2014-2-6.

Sendler, Ulrich Baum, Gerhard; Borcherding, Holger; Broy, Manfred; Eigner, Martin; Huber, Anton Sebastian; Kohler, Herbert K.; Russwurm, Siegfried; Stümpfle, Matthias (Hg.) (2013): Industrie 4.0. Beherrschung der industriellen Komplexität mit SysLM. Berlin: Springer Vieweg.
Wickett, Karen M.; Isaac, Antoine; Fenlon, Katrina S.; Doerr, Martin; Meghini, Carlo; Palmer, Carole L.; Jett, Jacob (2013): Modeling Cultural Collections for Digital Aggregation and Exchange Environments. Verfügbar unter https://www.ideals.illinois.edu/bit stream/2142/45860/3/EDM-DCC_Whitepaper_Final20131009. pdf.

Wissenschaftsrat (Hg.) (2011): Empfehlungen für Forschungsinfrastrukturen in den Geistes- und Sozialwissenschaften. Berlin. S. 67-71. Verfügbar unter http://www.wissenschaftsrat.de/dow nload/archiv/10465-11.pdf.

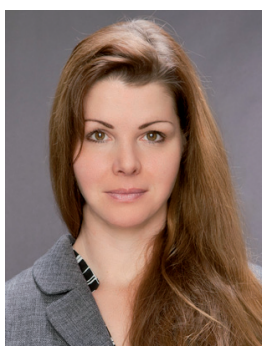

\section{Jenny Oltersdorf}

Fachhochschule Potsdam

Fachbereich Informationswissenschaften

Kiepenheuerallee 5

D-14469 Potsdam

oltersdorf@fh-potsdam.de

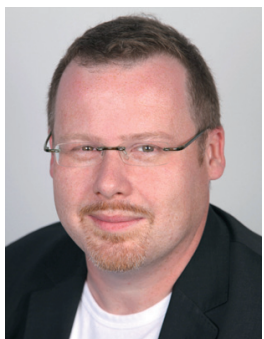

\section{Stefan Schmunk}

Niedersächsische Staats- und Universitätsbibliothek Göttingen Abteilung Forschung und Entwicklung Platz der Göttinger Sieben 1 D-37073 Göttingen schmunk@sub.uni-goettingen.de 